ISSN 1991-8631

Original Paper

http://indexmedicus.afro.who.int

\title{
Essai d'amélioration de la durée de conservation des aubergines aux températures ambiantes au Gabon
}

\author{
Alexis Nicaise LEPENGUE ${ }^{1 *}$, Isaac MOUARAGADJA ${ }^{1}$, Emmanuel DICK $^{2}$, \\ Bertrand MBATCHI ${ }^{1}$ et Séverin AKE ${ }^{2}$ \\ ${ }^{1}$ Université des Sciences et Techniques de Masuku, (USTM) Unité de Recherche Agrobiologie ; \\ BP : 067 Franceville, Gabon; Tel : (00242) 067647 38/ 07684362. \\ ${ }^{2}$ Université de Cocody-Abidjan, Laboratoire de Physiologie végétale, Agrophysiologie ; \\ 22 BP : 582 Abidjan 22. \\ *Auteur correspondant, E-mail: lepengue_nicaise@yahoo.fr
}

\section{RESUME}

La conservation des fruits, particulièrement celle des aubergines, est difficile au Gabon, par manque de techniques d'entreposage appropriées. Les rares procédés utilisés sont coûteux et peu accessibles à la majeure partie de la population. La présente étude a été proposée pour tester l'efficacité de quelques méthodes simples susceptibles de servir d'alternative. Ce sont: les traitements des aubergines à la poudre des cendres et aux solutions de GA-3, de $\mathrm{NaCl}$ et d'huile d'arachide. Les paramètres de conservation étudiés étaient : la durée de stockage, les pertes de masse, de dureté et d'intégrité des organes. Les résultats ont montré que les traitements à l'huile d'arachide produisaient les meilleures conditions de conservation, avec des rallongements significatifs de durée de +18 jours, et des atténuations des pertes de masse, de dureté et d'intégrité. L'usage de cette technique peut donc être conseillé comme méthode de conservation valable des fruits d'aubergine.

(C) 2012 International Formulae Group. All rights reserved.

Mots clés : Fruits, traitements, huile d'arachide, durée, stockage.

\section{INTRODUCTION}

L'aubergine africaine (Solanum aethiopicum L.) est une plante arbustive de la famille des Solanaceae. Elle est cultivée dans toute la zone tropicale d'Afrique, d'Amérique Latine et d'Asie, pour l'exploitation de ses fruits comestibles (Chen et al., 2000). Ce sont des baies globuleuses ellipsoïdales, ovoïdes ou fusiformes, possédant également des vertus thérapeutiques: sédative, carminative, anti colique, antibactérienne, anti hypertensive, anti tétanique (Lépengué et al., 2010), etc.
Le principal producteur africain d'aubergines est la Côte d'Ivoire, avec 60.000 tonnes enregistrées en 2000 (Lester et Seck, 2004). Au Gabon, l'aubergine constitue l'un des principaux fruits-légumes du pays. Sa culture s'effectue sur toute l'étendue du territoire, avec des techniques globalement traditionnelles. Si l'ensemble des productions périurbaines et rurales du pays parvient à satisfaire pleinement la demande locale, les méthodes de conservation laissent à désirer. En effet, le pays ne dispose d'aucun procédé de conservation fiable, susceptible de garantir, 
en période de contre saison, ou durant toute l'année, la disponibilité des fruits d'aubergines frais (Lépengué et al., 2010). Les rares techniques de conservation rencontrées sont majoritairement artisanales et basées sur la déshydratation des organes par voie de séchage ou fumage. Or, ces méthodes provoquent non seulement la perte de nombreux composés volatiles ou thermosensibles, mais affectent également la qualité organoleptique des fruits; ce qui abaisse fortement leur valeur marchande. Les méthodes conventionnelles en vogue (atmosphère contrôlée, usage d'adjuvants, conditionnement frigorifique etc.) restent assez onéreuses, et inaccessibles à la majorité des consommateurs gabonais (Lépengué et al., 2010). C'est pour résoudre cette problématique que la présente étude a été proposée. Elle vise à déterminer des procédés de conservation efficaces, simples, pratiques, peu coûteux et accessibles à la majorité des populations locales. Les techniques testées sont : l'immersion des fruits d'aubergine dans l'huile d'arachide, leur prétraitement dans les solutions de gibbérelline (GA-3) ou de sel de cuisine $(\mathrm{NaCl})$, et l'enrobage dans les poudres de cendre. L'efficacité de chaque traitement a été mesurée par la variation de 4 paramètres biochimiques, à savoir, la durée de conservation des fruits, la perte de masse, la consistance de l'épiderme et l'intégrité tissulaire des organes.

\section{MATERIEL ET METHODES Matériel}

Le matériel végétal utilisé dans cette étude est l'aubergine (Solanum aethiopicum L., Solanaceae). Les fruits de cette plante ont été récoltés dans les champs paysans du village Diénga Lewa Passo (latitude 1.85083; longitude 12.67667), dans la province de l'Ogooué Lolo, au Sud-Est du Gabon.

\section{Méthodes \\ Traitement des aubergines}

Les fruits d'aubergine étudiés dans ce travail ont été récoltés manuellement au champ. Ils ont précautionneusement été transportés au laboratoire $\left(25^{\circ} \mathrm{C}\right)$, préservés de tous chocs et blessures (Lépengué et al., 2010). Les fruits d'environ $30 \mathrm{~g}$ (sans pédoncule) ont été sélectionnés et désinfectés par trempage pendant $5 \mathrm{~min}$ dans une cuve contenant 50 L d'hypochlorite de sodium (1\%). Ils ont ensuite été desséchés entre 2 épaisseurs de papier buvard, et soumis à divers traitements (immersions respectives pendant 5 min dans $1 \mathrm{~L}$ d'huile d'arachide, 1 $\mathrm{L}$ de $\mathrm{NaCl} 1 \%$ ou $1 \mathrm{~L}$ de GA-3 $1 \%$, enrobage dans $1 \mathrm{~L}$ de poudre de cendre) (Lépengué et al., 2010).

Pour chaque traitement, 3 aubergines ont été utilisées, ce qui correspond à 12 organes pour les 4 traitements effectués. Trois aubergines non traitées ont également été conservées, mais dans les conditions ambiantes du laboratoire, pour servir de témoins. Tous les 5 jours (et pendant 30 jours), un organe a été retiré et soumis aux analyses morphométriques et biochimiques décrits ci-dessous.

\section{Evaluation des paramètres morphologiques et biochimiques : \\ Durée de conservation}

La durée de conservation des aubergines a été définie comme le temps s'écoulant entre le début d'incubation et l'apparition des premiers symptômes de nécrose tissulaire (taches de noircissement des peaux des organes) (Lépengué et al., 2010).

\section{Perte de masse}

Pour chaque organe, la perte de masse (\% $\mathrm{M})$ des aubergines a été déterminée après pesée des organes tous les 5 jours à l'aide d'une balance (Ohaus Analytic 60, USA), par la formule suivante (Lépengué et al., 2010) :

$\% M=\frac{M L-M L}{M t} \times 100$

Où Mi correspond à la masse initiale de l'aubergine et Mt, à la masse de l'organe au temps défini.

\section{Perte de résistance mécanique}

La perte de résistance mécanique des aubergines a été mesurée par application d'un 
pénétromètre de type arbalète, sur la partie médiane des fruits (Lépengué et al., 2010). Elle a été calculée à chaque instant par analogie à l'équation [1], sur la base des résistances initiale et finale.

\section{Perte d'intégrité}

Pour mesurer la perte d'intégrité des aubergines, 10 rondelles (diamètre $5 \mathrm{~mm}$; longueur $10 \mathrm{~mm}$ ) de chaque organe ont été prélevées à l'aide d'un emporte-pièce, immergées dans $10 \mathrm{ml}$ d'eau distillée désionisée, et incubées en agitation continue (agitateur Vibramax) pendant $1 \mathrm{~h}$ (Lépengué et al., 2008). Les solutions ont ensuite été filtrées sur papier Whatman $\mathrm{n}^{\circ} 2$ et leurs conductivités mesurées à l'aide d'un conductimètre de type Jenway 3410 Electrochemestry Analyser. Les pertes de conductivité ont été calculées à partir des valeurs initiales, suivant le modèle de l'équation [1].

\section{Analyse statistique}

Chacune des expériences décrites dans ce travail a été répétée 3 fois, et les résultats soumis à une analyse de variance au logiciel Statistica 6.0, au seuil de 5\%. Les tests comparatifs de Newman-Keuls ont été utilisés, en cas de différence significative.

\section{RESULTATS}

Effet des traitements sur la durée de conservation des aubergines

Les résultats du traitement des aubergines à la gibbérelline (GA-3), au chlorure de sodium $(\mathrm{NaCl})$, à la cendre et à l'huile d'arachide ont été présentés à la Figure 1. Leur analyse a révélé que ces 4 traitements allongeaient la durée de conservation des organes étudiés, par comparaison aux aubergines témoins. Les allongements les plus faibles ont été observés avec la GA-3 (+2 j) et le $\mathrm{NaCl}(+5 \mathrm{j})$, et les augmentations les plus fortes produites par la cendre $(+11 \mathrm{j})$ et l'huile d'arachide (+18 j). Quoique tous les traitements aient induit des augmentations de la durée de conservation des aubergines, les effets statistiquement significatifs n'ont été produits que par les cendres et l'huile d'arachide.

\section{Effet des traitements sur la perte de masse}

La pesée des aubergines préalablement traitées à la gibbérelline, au chlorure de sodium, à la cendre et à l'huile d'arachide a donné les résultats présentés à la Figure 2 . Leur examen a clairement ressorti que les 4 traitements modéraient les pertes de masse des organes étudiés. A la fin de l'étude au $30^{\mathrm{e}}$ jour, les réductions de masse se sont révélées fortes pour les traitements à la gibbérelline $(28 \%)$ et au chlorure de sodium $(22 \%)$, et assez proches du témoin (36\%). Les effets statistiquement significatifs n'ont donc été produits que par les traitements aux cendres et à l'huile d'arachide, avec des taux de réduction respectifs de $16 \%$ et $10 \%$ à la même durée de stockage.

Effet des traitements sur la résistance mécanique des aubergines

La Figure 3 présente les résultats de l'effet de différents traitements de conservation des aubergines sur la résistance mécanique desdits organes. Tous les traitements ont présenté de bonnes aptitudes à maintenir intacte la fermeté des organes étudiés pendant les 30 jours de stockage. Les meilleurs résultats ont été produits par les cendres et l'huile d'arachide avec des pertes de résistance mécanique respectives de $14 \%$ et $40 \%$ au $30^{\mathrm{e}}$ jour. Les pertes de dureté provoquées par les traitements à la gibbérelline $(80 \%)$ et au chlorure de sodium $(60 \%)$ n'ont pas statistiquement été différentes de celles produites par les conditions naturelles $(97 \%)$, à la même durée de conservation.

Effet de différents traitements sur l'intégrité tissulaire des aubergines

Les résultats de l'impact de différents traitements de conservation des aubergines sur l'intégrité cellulaire de ces tissus ont été présentés à la Figure 4. Leur analyse a clairement révélé que tous les 4 traitements 
testés freinaient diversement la perte d'intégrité des aubergines. Les effets de la GA-3 (76\%) et du $\mathrm{NaCl}(63 \%)$ ne se sont pas révélés significatifs, par rapport au témoin (96\%), à la fin de l'expérimentation, au $30^{\mathrm{e}}$ jour. Les meilleurs résultats ont alors été obtenus avec la cendre et l'huile d'arachide, dont les taux de perte (respectivement de $12 \%$ et $15 \%$ ) se sont révélés significatifs, au bout de 30 jours d'expérimentation.

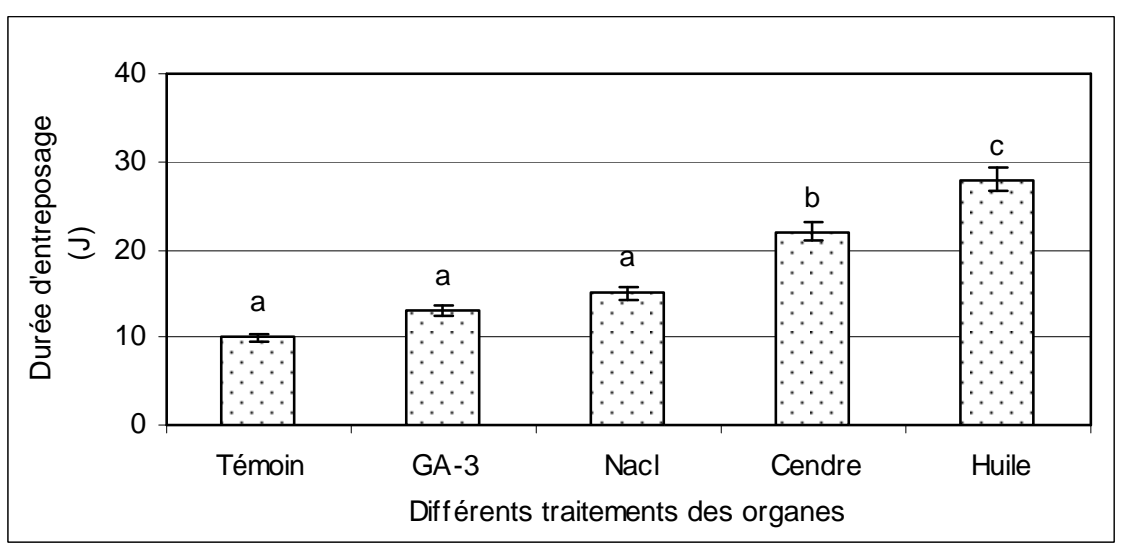

Les durées de conservation surmontées de lettres identiques ne sont pas significativement différentes au seuil de $5 \%$.

Figure 1: Effet de 4 traitements sur la durée de conservation des fruits d'aubergine à la température de $25^{\circ} \mathrm{C}$.

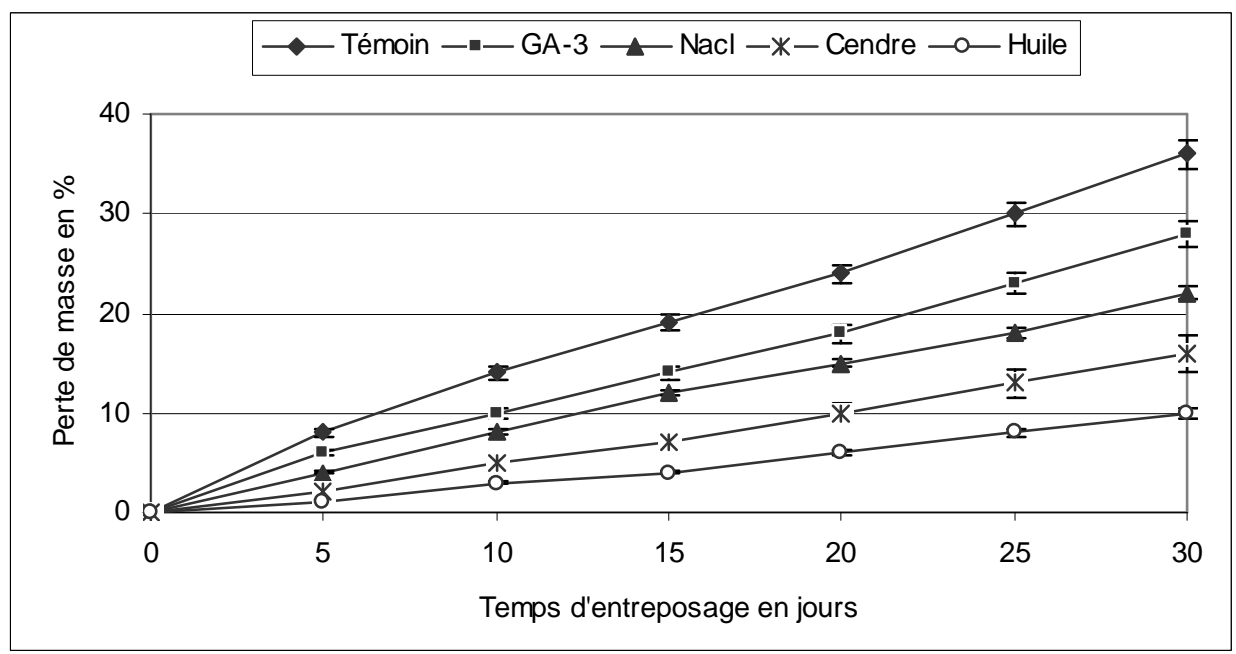

Figure 2: Evolution des pertes de masse durant le stockage des fruits d'aubergine préalablement soumis à divers traitements de conservation à la température de $25^{\circ} \mathrm{C}$. 


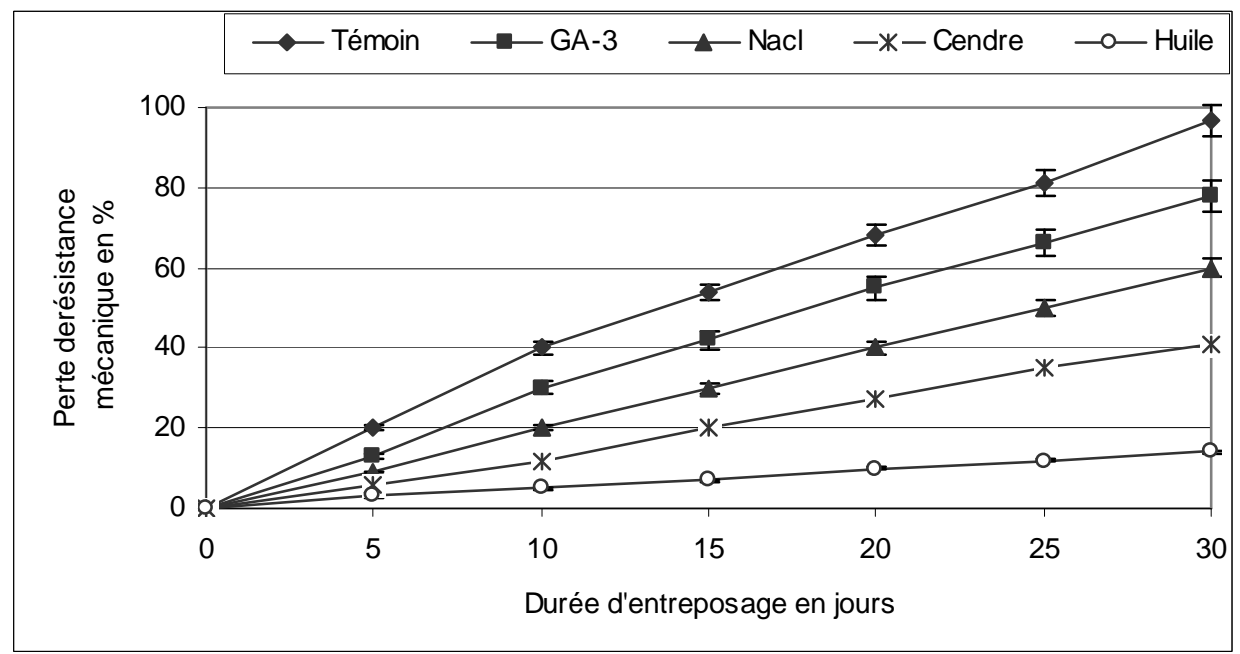

Figure 3 : Evolution de la résistance mécanique, durant le stockage, des fruits d'aubergine préalablement soumis à divers traitements de conservation à la température de $25{ }^{\circ} \mathrm{C}$.

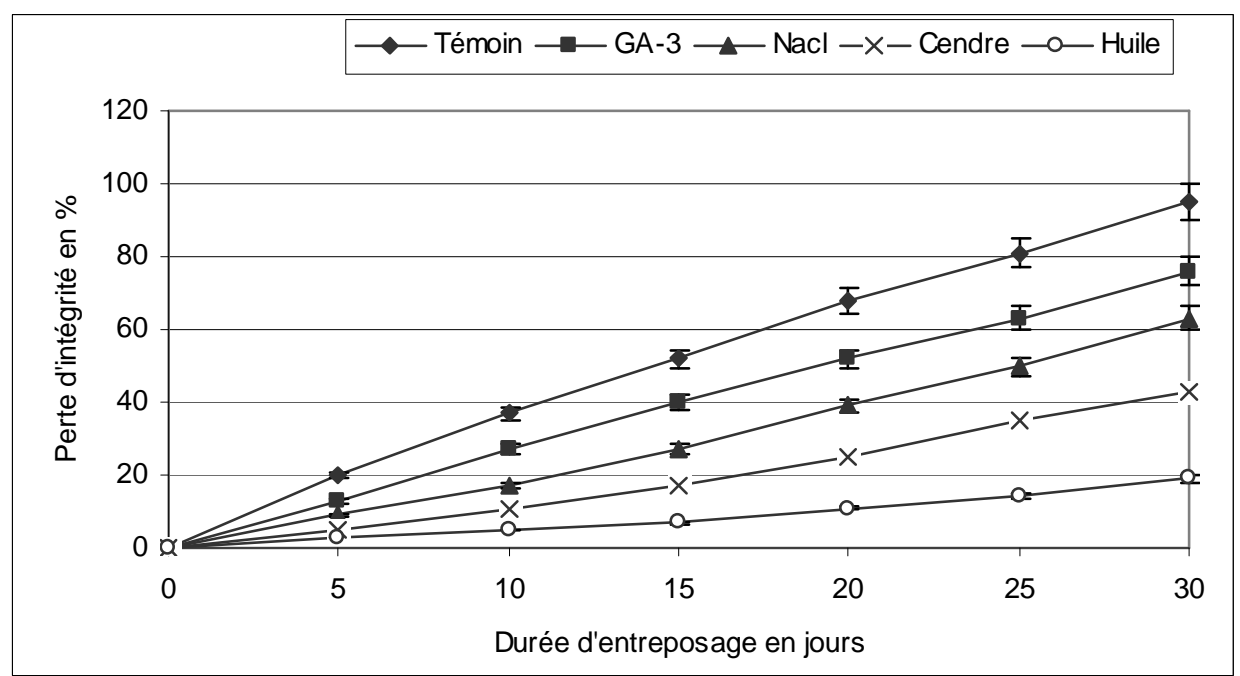

Figure 4 : Perte d'intégrité, au cours du stockage, des fruits d'aubergine préalablement soumis à divers traitements de conservation à la température de $25^{\circ} \mathrm{C}$.

\section{DISCUSSION}

Les résultats de cette étude ont montré que tous les traitements testés augmentaient la durée de conservation des aubergines, et limitaient l'ampleur des pertes de masse, de consistance et d'intégrité tissulaire. Des résultats similaires ont déjà été signalés par Dick (2006) et Lépengué et al. (2010) sur les bananes plantain cultivar Corne I conservées à la température de $25^{\circ} \mathrm{C}$. 
D'une façon générale, l'efficacité de la conservation des fruits dépend fortement des conditions de stockage de ces organes (Varoquaux et al., 2002). Globalement, les températures, les pressions élevées et l'abondance d'oxygène accélèrent la dégradation des fruits, alors que les faibles mesures de ces 3 paramètres allongent la durée de vie verte des organes. Chez les dattes dorées par exemple, Moshen et Naoufel (2005) ont montré que cette durée pouvait être rallongée de plusieurs jours dans les conditions de température correspondant à 20 ${ }^{\circ} \mathrm{C}$, et d'oxygène appauvri aux taux de $1 \%$. Des résultats semblables ont également été signalés sur les pêches et les fraises aux conditions de température et d'oxygène correspondant respectivement à $18{ }^{\circ} \mathrm{C}$ et $2 \%$ (Leitsner et Gould, 2002).

Le prolongement de la durée de vie verte des aubergines dans notre étude laisse penser que les différents traitements appliqués modifient favorablement les interactions des fruits avec l'environnement ambiant. En tenant compte de la nature et de la réactivité des produits testés, on peut supposer que les différentes actions de ces produits sont de types physiques ou biochimiques, comme l'ont suggéré Narayana et al. (2002) et Varoquaux et al. (2002).

La forme solide des cendres utilisées et la fluidité de l'huile d'arachide militent en faveur d'une action physique, par constitution probable d'un revêtement superficiel à l'épiderme des aubergines. Ces dépôts extérieurs représenteraient ainsi des barrières qui limitent des échanges avec le milieu ambiant. Les impacts de la température, de la pression et de l'oxygène seraient alors réduits, créant ainsi des sortes d'enceintes d'atmosphères contrôlées, conduisant au prolongement de la vie verte des organes. Des résultats similaires ont déjà été signalés sur les bananes par Chamara et al. (2000), Chen et al. (2000) et Karunaratne et al. (2002).

Les actions du $\mathrm{NaCl}$ et de la GA-3 semblent être essentiellement physiologiques. Les observations visuelles (non présentées) et à la loupe montrent en effet que ces composés ne persistent pas sur les épidermes des organes après trempage. Ces éléments pourraient donc agir par obstruction ou resserrement des espaces et interstices épidermiques, limitant ainsi les échanges entre les fruits et le milieu ambiant (Angels et al., 2000).

Dans le cas du $\mathrm{NaCl}$ par exemple, les travaux de El Jaafari (1993) ont montré que la salinité engendrait la formation des agrégats au niveau des solutions nutritives du sol. Il en résultait une cimentation du milieu, entravant fortement les phénomènes de succion racinaire, et aboutissant au flétrissement des plantes (Rajaskaran et al., 2000).

Dans les zones épidermiques des aubergines, des phénomènes similaires sont susceptibles de se produire. Les compartiments cytosoliques riches en solutés (Heller et al., 2006) pourraient réagir en formant des agrégations tissulaires, aboutissant à l'obstruction ou à la réduction de la perméabilité des organes. Ces faits limiteraient donc les échanges entre les fruits et le milieu extérieur, conduisant au rallongement de la durée de vie des organes.

\section{Conclusion}

Le traitement des fruits d'aubergine au $\mathrm{NaCl}$, à la $\mathrm{GA}-3$, à la cendre et à l'huile d'arachide freine les pertes de leur consistance, et augmente leur durée de conservation. Les effets les plus significatifs sont produits par les traitements des fruits à l'huile d'arachide. Dans les pays qui ne disposent d'aucune technique fiable de conservation des fruits et légumes et dont les enceintes réfrigérées sont peu répandus, les techniques d'immersion à l'huile d'arachide peuvent donc constituer des alternatives valables pour un usage domestique d'appoint.

\section{REFERENCES}

Angels BM, Delamor F, Amaros A, Serrano M, Martinez V, Cerda A. 2000. Polyamines ethylene and other physico chemical parameter in tomato (Lycopersicum esculentum) fruits as 
affected by salinity. Physiologia Plantarium, 109: 428-434.

Chamara D, Illeperuma K, Theja Galappatty P, Sarananda KH. 2000. Modified atmosphere packaging of 'Kolikuttu' bananas at low temperature. Journal of Horticultural Science and Biotechnology, 75(1): 92-96.

Chen W, Wu Z, Su M, Zhu J. 2000. Technologie post-récolte, transport et commercialisation des bananes en Chine. Agrociencia, 36(2): 169-180.

Dick E. 2006. Contribution à l'étude de la physiologie du mûrissement, de l'entreposage et de la conservation de la banane plantain après récolte. Thèse d'Etat, Université de Cocody-Abidjan, $243 \mathrm{p}$.

El Jaafari S. 1993. Contribution à l'étude des mécanismes biophysiques et biochimiques de résistance à la sécheresse chez le blé. Doctorat, Faculté des Sciences Agronomiques de Gembloux, Belgique, 214p.

Heller R, Esnault R, Lance C. 2006. Physiologie Végétale. Développement $\left(6^{\mathrm{e}}\right.$ édition de l'abrégé). Editions Dunod: Paris ; 366 p.

Karunaratne AM, Ethugala CV. 2002. État sanitaire, changements physicochimiques et goût des bananes traitées avec de l'acide acétique ou du vinaigre. Fruits, 57(1): 11-18.

Leitsner P, Gould J. 2002. Technologies combinées de conservation des fruits et des légumes. Archives de Documents de la FAO. www.fao.org/docrep/006/ J1954F/J1954f04.htm
Lépengue AN, Mouaragadja I, Dick E, M'batchi B, Aké S. 2010. Amélioration de la durée de conservation des bananes plantains aux températures ambiantes. Int. J. Biol. Chem. Sci., 4(3): 730-737.

Lépengué AN. 2008. Contribution à la protection de la roselle (Hibiscus sabdariffa L. var. sabdariffa), contre la pourriture engendrée par Phoma sabdariffae Sacc. et Trichosphaeria sp., au Gabon : Etude des mécanismes d'action fongiques phytotoxiques. Doctorat d'Université, UFR Biosciences, Univ. Cocody-Abidjan, 294 p.

Lester RN, Seck A. 2004. Solanum aethiopicum L. In Légumes. Edition PROTA 2: Waningen, Pays Bas ; $306 \mathrm{p}$.

Moshen A, Naoufel B. 2005. Effet des conditions d'entreposage sur la dégradation de la couleur des dattes tunisiennes de type Deglet-Nour. Fruits, 60(1): 41-46.

Narayana CK, Mustafa MM, Sathiamoorthy S. 2002. Influence de l'emballage et du stockage sur la durée de vie et la qualité des bananes de la variété Karpuravalli. Indian Journal of Horticulture, 59(2): 113-117.

Rajaskaran LR, Aspinal D, Paleg LG. 2000. Physiological mechanism of tolerance of Lycopersicum spp. exposed to salt stress. Canad. J. Plant Sci., 80(1): 151-152.

Varoquaux P, Gouble B, Ducamp MN, Self G. 2002. Méthode permettant d'optimiser l'emballage des fruits sous atmosphère modifiée. Fruits, 57(5-6): 313-322. 